\title{
UAVS ENHANCED NAVIGATION IN OUTDOOR GNSS DENIED ENVIRONMENT USING UWB AND MONOCULAR CAMERA SYSTEMS
}

\author{
S. Zahran ${ }^{\text {a, }}{ }^{*}$, A. Masiero ${ }^{\text {b }}$, M.M. Mostafa ${ }^{\text {a }}$, A. M. Moussa ${ }^{\text {a,c }}$, A. Vettore ${ }^{\text {b }}$, N. El-Sheimy ${ }^{\text {a }}$ \\ ${ }^{a}$ Department of Geomatics, University of Calgary, Calgary, Canada - (shady.zahran1, mostafa.mostafa, elsheimy)@ucalgary.ca \\ ${ }^{\mathrm{b}}$ Interdepartmental Research Center of Geomatics (CIRGEO), University of Padova, \\ Viale dell’Università 16, Legnaro (PD) 35020, Italy - (masiero@ dei.unipd.it , antonio.vettore@ unipd.it) \\ c Department of Electrical Engineering, Port-Said University, Port Said, Egypt - amelsaye@ ucalgary.ca
}

Commission I, ICWG I/II

KEY WORDS: Optical Flow, Ultra-Wideband, Multi-Sensor Fusion, Inertial Navigation Systems, Multi-Sensor Fusion, Global Navigation Satellite System Denied Environment.

\begin{abstract}
:
The demand for small Unmanned Aerial Vehicles (UAVs) is massively increasing these days, due to the wide variety of applications utilizing such vehicles to perform tasks that may be dangerous or just to save time, effort, or cost. Small UAVs navigation system mainly depends on the integration between Global Navigation Satellite Systems (GNSS) and Inertial Measurement Unit (INS) to estimate the Positions, Velocities, and Attitudes (PVT) of the vehicle. Without GNSS such UAVs cannot navigate for long periods of time depending on INS alone, as the low-cost INS typically exhibits massive accumulation of errors during GNSS absence. Given the importance of ensuring full operability of the UAVs even during GNSS signals unavailability, other sensors must be used to bound the INS errors and enhance the navigation system performance. This paper proposes an enhanced UAV navigation system based on integration between monocular camera, Ultra-Wideband (UWB) system, and INS. In addition to using variable EKF weighting scheme. The paper also investigates this integration in the case of low density of UWB anchors, to reduce the cost required for such UWB system infrastructure. A GoPro Camera and UWB rover were attached to the belly of a quadcopter, an on the shelf commercial drone (3DR Solo), during the experimental flight. The velocity of the vehicle is estimated with Optical Flow (OF) from camera successive images, while the range measurements between the UWB rover and the stationary UWB anchors, which were distributed on the field, were used to estimate UAV position.
\end{abstract}

\section{INTRODUCTION}

The demand of versatile small UAVs is increasing rapidly, due to the massive spread of applications that utilize such UAVs to accomplish different tasks in order to save cost, effort, time, or tasks that may expose people to danger (Surveillance \& Reconnaissance, inspection \& monitoring, survey \& mapping, industrial inspection, maintenance \& repair of oil \& gas platforms'. Etc). Such wide range of applications that utilize UAVs in different circumstances, areas, or conditions, makes the development of navigation systems effective in all such operative conditions essential for ensuring safe and proper use of UAVs in critical environments.

The current generation of UAVs navigation systems mainly depend on the fusion between the measurements of GNSS and INS through filters to be able to navigate for long and short time periods (Noureldin et al., 2013). Typically, the Inertial Measurement Unit (IMU) used in this kind of UAVs (small/commercial) is based on low-cost Micro-ElectroMechanical Systems (MEMS). Since this kind of sensor typically accumulates a huge amount of errors during the navigation estimation process (mechanization), they can be used as standalone navigation solutions only for short time periods. Consequently, GNSS is typically used to bound INS errors, enabling the navigation system to correctly work for long time periods. Unfortunately, problems arise once the GNSS system is jammed, blocked, or spoofed: in such cases, an alternative aiding system should be used to replace the GNSS in order to ensure a versatile navigation system.

Different sensors were considered in the literature to properly bound INS errors. First, a standard camera is often considered to this aim, mostly because of its small size, lightweight, and low power consumption, which are adequate characteristics for small UAVs. Furthermore, camera sensor is often mounted on UAVs also for other purposes. Visual Odometry (VO) is one of the most common vision-based techniques for estimating the vehicle velocity: in $\mathrm{VO}$, the estimation of the vehicle motion is computed analyzing successive camera images, i.e. by detecting features/textures matched in two successive frames (Yousif et al., 2015). Depending on the number of available cameras, VO can be monocular or stereo. Furthermore, it can be based on different image processing techniques: optical flow (Mansur et al., 2017), features matching (Chuanqi et al., 2017), or feature tracking (Johnson et al., 2008). (Mostafa et al., n.d.) proposed a novel approach based on integrating a monocular camera, IMU, and Artificial Intelligent (AI) through EKF, where the AI is used to estimate the underlying function of the camera and diminish the need for estimating the geometric camera model. Since monocular camera approaches typically suffer from scale estimation issues, such approaches usually integrate the camera information with that provided by other sensors, for instance, the barometer (Nistér et al., 2004). Alternatively, scale estimation issues can be tackled by using a stereo-camera, such as in (Eynard et al., 2010), where the authors assumed a plane ground, and they utilized a fisheye camera and a respective camera: the fisheye

${ }^{*}$ Corresponding author. 
camera provided a wide field of view, whereas the other one provided a higher measurement accuracy. Despite the potential of stereo-camera approaches is typically higher than that of monocular ones, the capabilities of stereo-camera systems mounted on small UAVs are quite limited because of the very short baselines that can be used in such cases. Actually, cameras are very adequate for small drones but their sensitivity to environmental changes may affect their performance and cause errors in navigation.

Ultra-wideband sensors are mainly used for indoor terrestrial applications, but there are several recent types of research that considered this kind of technology for UAV navigation purposes. In (Tiemann et al., 2015), the authors run a real indoor experiment with a quadcopter and UWB mounted on it, to assess UWB system accuracy in a real indoor application. Other researches adopted UWB for precise Unmanned Aircraft Systems (UAS) autonomous landing (Kong et al., 2015). Furthermore, (Tiemann and Wietfeld, 2017) integrated the Time Difference of Arrival (TDOA) through a tightly coupled scheme in a constrained indoor environment using three UAVs. The low power consumption and lightweight of UWB devices make them adequate to be used with small drones. However, UWB devices usually cover only a relatively small area, hence a large UWB network can be required in outdoor UAV applications, consequently resulting in quite expensive costs to cover large areas.

This paper proposes the integration between a camera and a UWB system for outdoor UAV navigation when GNSS is not available, with variable weighting uncertainties EKF scheme. Also, this integration is investigated when the size of the UWB network is decreased by $33 \%$ in order to reduce the overall cost of the system.

\section{SYSTEM OVERVIEW}

This work integrates the information coming from two systems, camera, and UWB. The camera is used as VO to estimate the velocity of the drone based on optical flow approach, although the velocity estimate from the camera greatly enhances the navigation solution, but the orientation of the drones is not observable, which will lead to orientation drift. Differently, the UWB system will be used as CUPT update for the drone, where such CUPT update will enhance the orientation estimation as well. Both systems measurements are fused through EKF but each with a variable uncertainty. The camera measurements uncertainty is estimated based on the percentage of outliers compared to inliers features in each successive camera frames. As if the inliers are more than the outliers we have more trust in the estimated velocity from the camera and vice versa. While in case of UWB system, the weighting is calculated based on the number of UWB anchor in view, in addition to comparing the estimated position from UWB against the position estimated from the mechanization process.

Since the use of an UWB-based drone position update in the outdoor environment requires a quite large, and hence expensive infrastructure, the integration with a camera system is investigated in this work. Such investigation allows reducing the size (and costs) of the UWB infrastructure while ensuring reliable estimates of the navigation unknowns for longer periods, as will be shown in Section 3 .

\subsection{Monocular Visual Odometry}

The camera is attached to the belly of the drone (nadir), the implemented VO is based on the optical flow approach shown in (Heinrich, 2017), where features are detected in consecutive frames (30 FPS) through the Speeded Up Robust Features (SURF) detector (Bay et al., 2006), which has been chosen because of its low computational requirements. Then, the matched features pass through an M-estimator Sample Consensus (MSAC) algorithm for outlier rejection purpose.

To estimate the forward vehicle velocity a pinhole camera model is employed to project a point in space to a point in the image plane as (1):

$$
p=\frac{f_{\text {cam }}}{h} P
$$

Where: $\quad \mathrm{P}$ is a point in space $=[\mathrm{X}, \mathrm{Y}, \mathrm{Z}]$.

$p$ is the projection of the point of interest in the camera frame $=\left[x_{\text {pix }}, y_{\text {pix }}, f_{\text {cam }}\right]$.

$f_{\text {cam }}$ is the camera focal length.

$\mathrm{h}$ height between the camera origin and the ground.

The difference between the positions of the matched features $(\Delta x$ and $\Delta y)$ in pixels in the camera frame is then convert to real world displacement ( $\Delta X$ and $\Delta Y$ ) in meters as shown in (2).

$$
\Delta X=-\frac{S}{f_{\text {cam }}} \Delta x \cdot h
$$

Where: $\quad S$ is the pixel size

In order to calculate the drone forward velocity, the optical flow vectors must be compensated for the drone rotation with the aid of gyro measurements $\left(w_{x, y, z}\right)$ as shown in (3).

$$
V_{F}=-\left[\frac{S}{f_{c a m}} \Delta x-f_{c a m} \tan \left(w_{y} \Delta t\right)\right] * h
$$

Where: $\quad V_{F}$ is the vehicle forward velocity. $\Delta t$ is the time between successive frames.

\subsection{UWB System}

The low-cost UWB positioning (Pozyx) system used in this work is composed of 7 devices: 6 stationary beacons (anchors), and one moving beacon (rover) that is mounted on the UAV. Despite their low cost might make their performance worse than that of higher cost UWB systems, their ease of configuration, development, scaling, lightweight and small size are clearly advantageous factors.

Ranges between UWB devices are calculated by means of Time of Flight (TOF) measurements of radio signals. If anchor locations are fixed and known, the rover position can be estimated through trilateration by exploiting the anchor-rover range measurements.

It is worth to notice that the best ranging performance of UWB devices is ensured when working in clear line of sight conditions (CLOS). While in indoor applications such working condition is often not guaranteed, in the case of UAV outdoor tracking CLOS measurements are quite common, hence ensuring good ranging performance of the UWB system. 


\subsection{EKF Scheme}

Both the velocity from the camera and positions from the UWB system where fused through a loosely couple EKF scheme. The EKF error state vector is composed of 21 states (4).

$$
\delta x=\left[\delta \mathrm{P}^{\mathrm{n}} \delta \mathrm{V}^{\mathrm{n}} \delta \varepsilon^{\mathrm{n}} A_{\text {bias }} G_{\text {bias }} A_{s f} G_{s f}\right]^{\prime}
$$

Where: $\delta \mathrm{P}^{\mathrm{n}}, \delta \mathrm{V}^{\mathrm{n}}, \delta \varepsilon^{\mathrm{n}}$ are the position, velocity and attitudes error vectors.

$A_{\text {bias }}$ and $G_{\text {bias }}$ are the accelerometers and gyros biases respectively.

$A_{s f}$ and $G_{s f}$ are the scale factor of the accelerometers and gyros.

The EKF workflow is composed of two steps, first, it starts with prediction step, which is obtained by linearizing the mechanization equations to yield the system model as shown in (5) and (6).

$$
\begin{gathered}
\dot{x}=F x+G w \\
\hat{x}_{k}^{-}=\Phi_{k, k-1} \hat{x}_{k-1}+G_{k-1} w_{k-1}
\end{gathered}
$$

Where: $x$ is the error states,

$\mathrm{F}$ is the dynamic matrix

$\Phi$ is the state transition matrix

$\mathrm{G}$ is the noise coefficient matrix

$w$ is the system noise.

The noise model utilized to represent the colored noise in the accelerometer biases and scale factors is first order GaussMarkov (GM).

The error state prediction step is then followed by state uncertainty prediction (covariance matrix prediction) as (7).

$$
P_{k}^{-}=\Phi_{k, k-1} P_{k-1} \Phi_{k, k-1}^{T}+G_{k-1} Q_{k-1} G_{k-1}^{T}
$$

Where: $P$ is the state covariance matrix

$Q$ is the system noise $(w)$ covariance matrix.

When there are measurements from an external aiding sensor, the update equations follow the prediction step, to fuse these measurements with the predicted one according to its uncertainties as shown in (8-10).

$$
\begin{gathered}
K_{k}=P_{k}^{-} H_{k}^{\mathrm{T}}\left(H_{k} P_{k}^{-} H_{k}^{\mathrm{T}}+R_{k}\right)^{-1} \\
\hat{x}_{k}=\hat{x}_{k}^{-}+K_{k}\left(Z_{k}-H_{k} \hat{x}_{k}^{-}\right) \\
P_{k}=\left(I-K_{k} H_{k}\right) P_{k}^{-}
\end{gathered}
$$

Where: $K_{k}$ is the Kalman gain.

$R_{k}$ is the covariance matrix of the measurements.

$H_{k}$ is the design matrix.

$Z_{k}$ is the observation matrix.

\subsection{Data Fusion}

The weights of the measurements coming from the camera inside the EKF are not constant: weights are determined according to the percentage of inliers with respect to the outlier features found in the currently considered frames.

Measurements from the UWB system are weighted according to the number of available measurements from the beacons and their relationship with the previously predicted position from the mechanization process. The work flow of the proposed approach is showed in Figure 1.

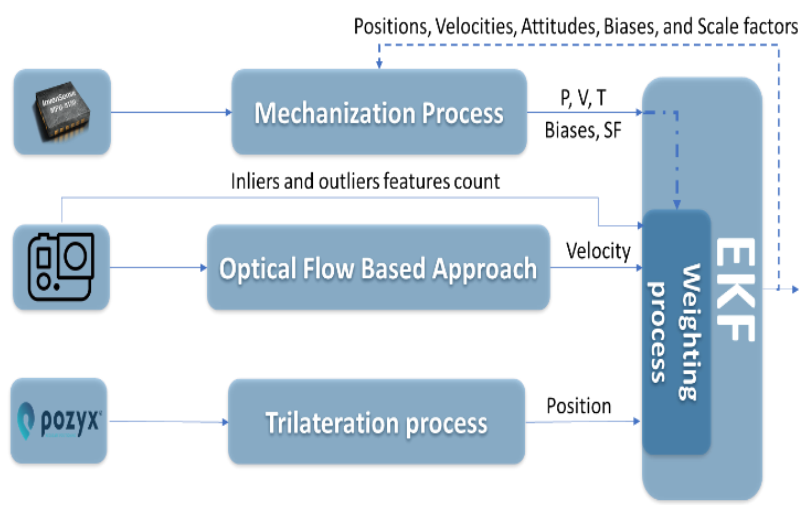

Figure 1. The work scheme of the proposed integration between camera and UWB system through Extended Kalman Filter loosely coupled scheme.

This variable weighting scheme of the UWB system is based on the existence of reliable velocity update, as with velocity update and low velocities of such small UAVs the error in the quadcopter position will be small within short period of time.

\subsection{Hardware Setup}

The drone used in this experiment is a commercial drone (3DR Solo) with low-cost MEMS-based IMU (MPU-9250), with a 5Hz- U-blox GPS. GoPro Hero 4 camera with a resolution of $1080 * 1920$, with 30 frames/secs in addition to the UWB rover is attached to the belly of the quadcopter as shown in Figure 2.

The 6 stationary beacons where approximately homogeneously distributed on the area of interest. The positions of the anchors were surveyed with a Trimble R10 GNSS receiver.

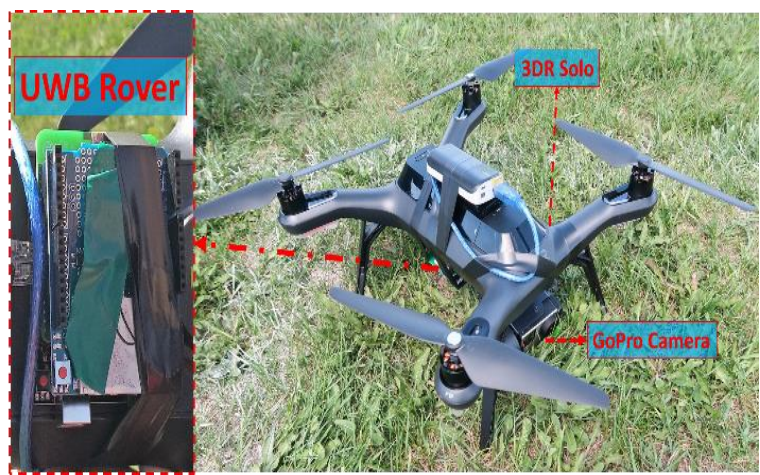

Figure 2.3DR Quadcopter with UWB system and camera attached to it.

\section{EXPERIMENT AND RESULTS}

The Flight extended for 186 seconds with 23 waypoints around the area of interest shown in Figure 3, the trajectory includes complex maneuvers to show the ability of the proposed approach to enhance the navigation performance during GNSS signal outage even during harsh movements.

Figure 4 shows the velocity estimated from the camera with the aid of optical flow approach compared to the reference velocity. The reference velocity was abstained from the onboard full navigation system (GNSS, INS, and magnetometer). 


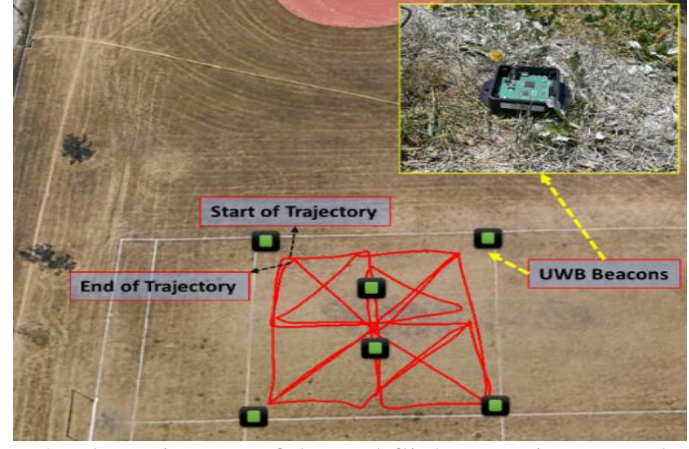

Figure 3. The trajectory of the real flight experiment, and UWB anchors distributed around the area of interest

Sample of the estimated position from the UWB system with $33 \%$ less in the infrastructure (4 anchors) compared to the positions estimated from the full UWB system is shown in
Figure 5 with six different non-homogenous configurations.

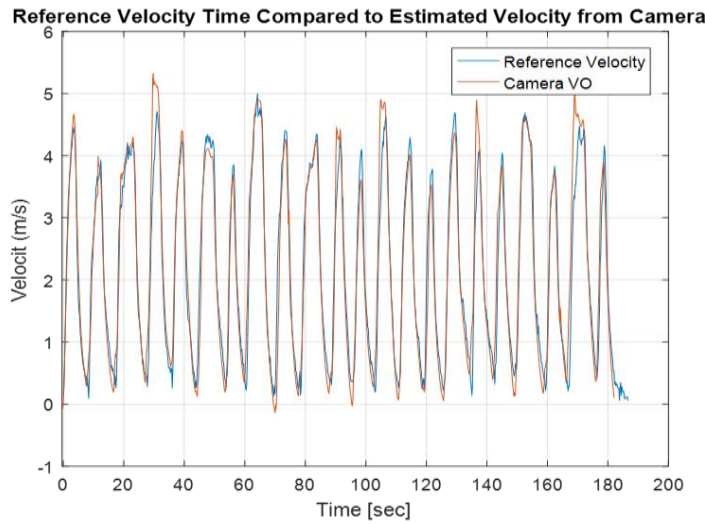

Figure 4. Velocity estimated from the camera using optical flow compared against reference velocity.

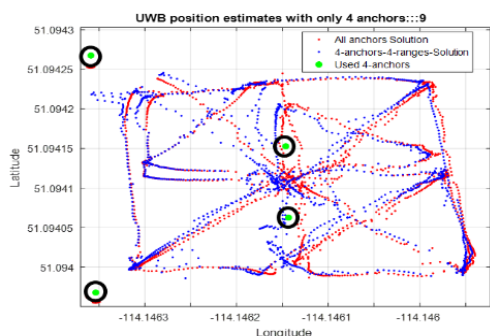

(a)

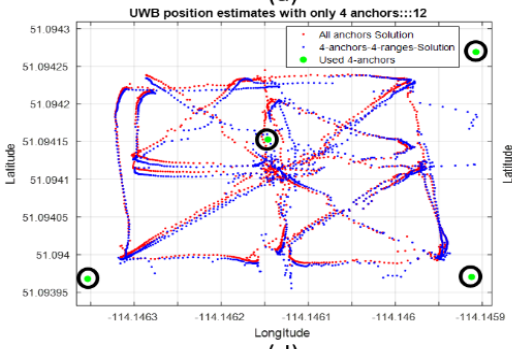

(d)

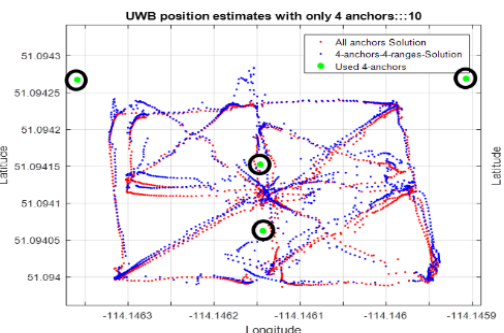

(b)

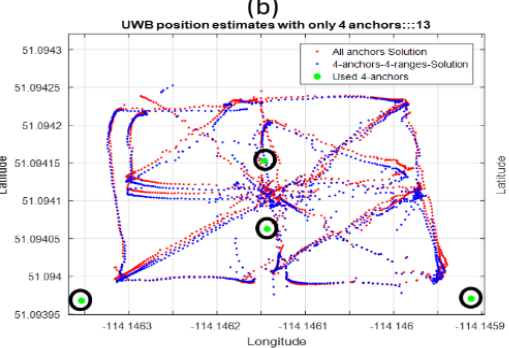

(e)
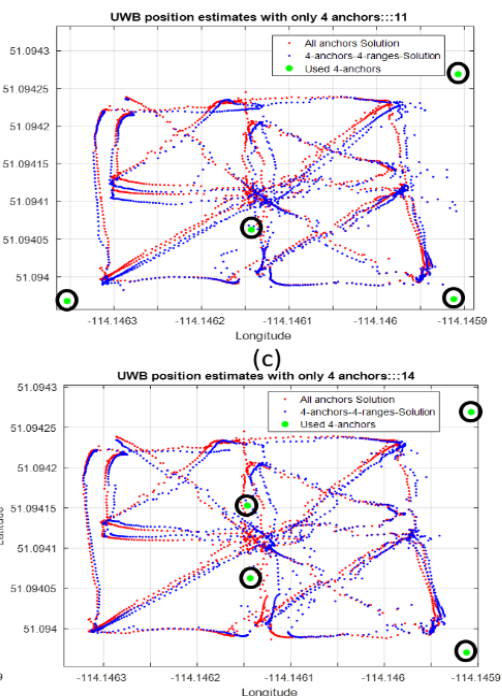

(f)

Figure 5. Sample of UWB system positions estimate when utilizing only 4 anchors compared to UWB system when utilizing 6 anchors

\subsection{IMU Stand-alone Solution (Dead-reckoning)}

The main purpose of this experiment is to show the inability of the low-cost IMU to estimate the navigation unknowns during the absence of GNSS signals as shown in Figure 6.

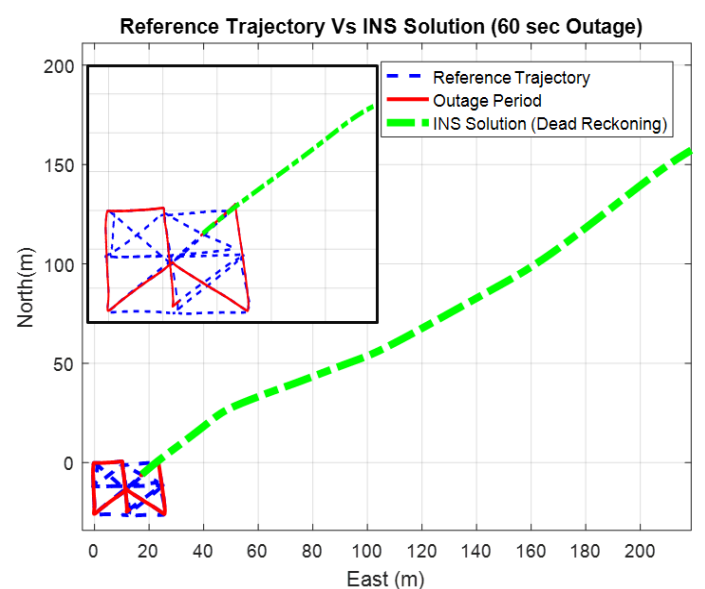

Figure 6. INS dead reckoning solution compared against reference trajectory for 60 secs of GNSS signals outage
The solution of the IMU as stand-alone system exhibits massive drift reached hundreds of meters in North and East direction (RMSE_N $250 \mathrm{~m}$, and RMSE_E $560 \mathrm{~m}$ ) during 60 seconds of GNSS outage.

\subsection{UWB-Camera-INS Integration (All Beacons Used)}

This experiment shows the performance of the UWB system alone when all the beacons are used as shown in Figure 7 and 8, while the performance of the camera integrated with INS alone is shown in Figures 9 and 10, and this experiment also proves the ability of the proposed integration between both systems to enhance the navigation solution even further despite using all the available UWB anchors as shown in Figures 11 and 12.

As shown in Figures 7 and 8 that in the outdoor environment, the performance of the UWB system with 6 anchors distributed around the designated area enhanced the performance of the navigation system in GNSS denied environment for 3 minutes, with RMSE error 1.4 and 1.2 meters in North and East direction respectively, compared to hundreds of meters when utilizing INS alone. 


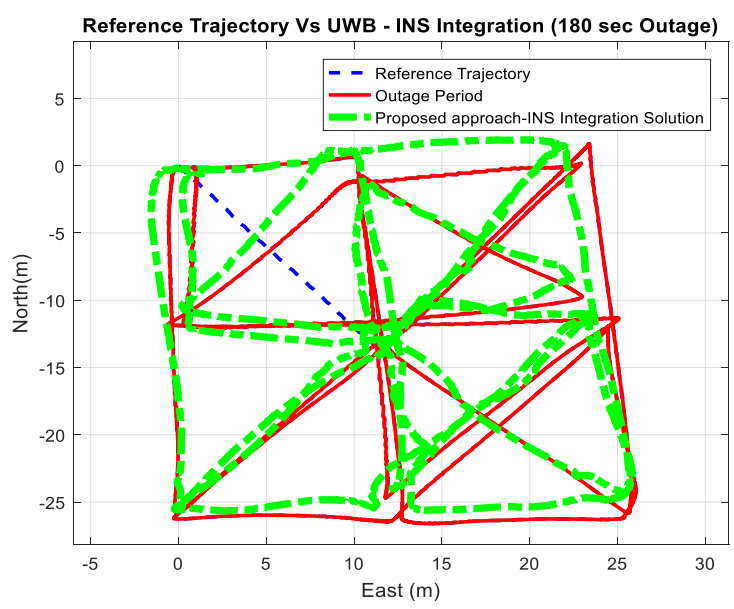

Figure 7. UWB-INS integration performance compared against reference trajectory for full GNSS Signal outage (3 minutes)

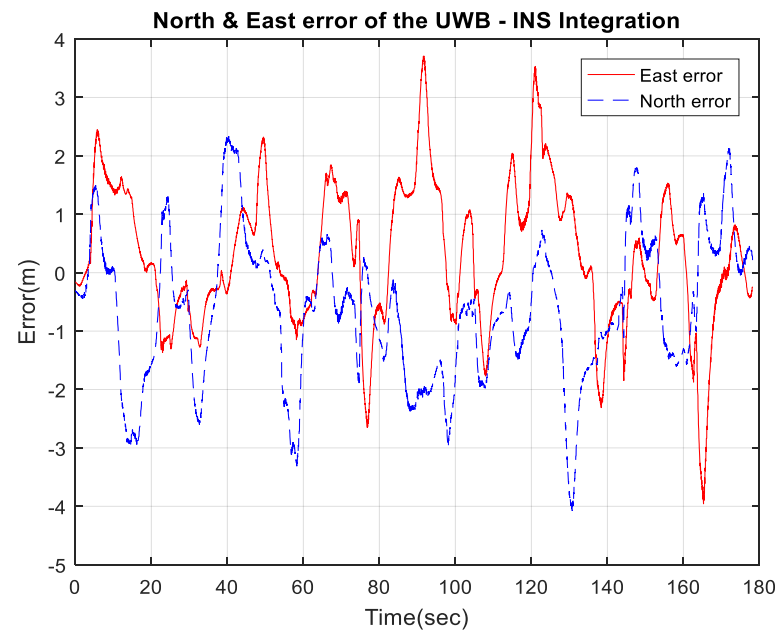

Figure 8. UWB-INS integration North and East errors for 180 seconds of complete GNSS signal outage

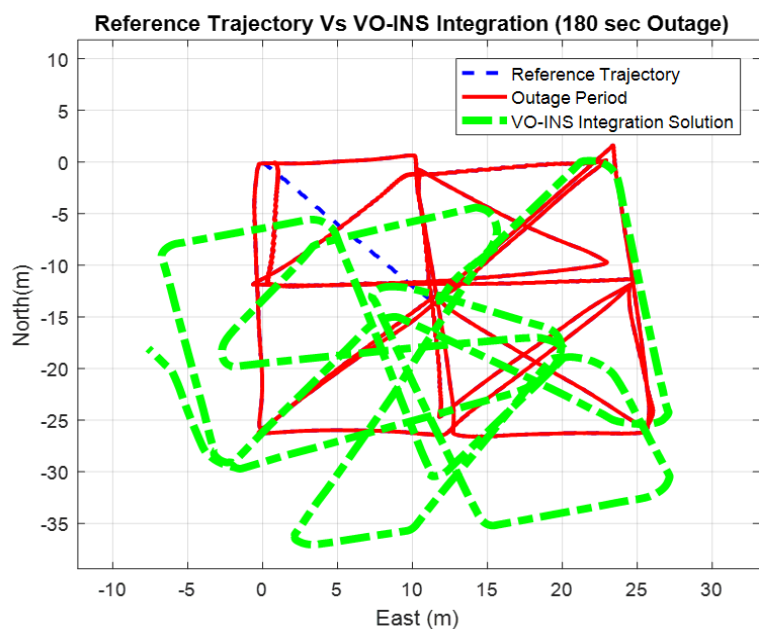

Figure 9. Performance of the integration between the camera and INS for 180 seconds of a complete signal outage

Figures 9 and 10 showed that the camera alone as aiding system enhanced the navigation performance, compared to INS deadreckoning solution, but it suffers from orientation drift and some links suffer from errors due to errors in camera velocity estimated which may be from some outliers which are not removed during MSAC process.

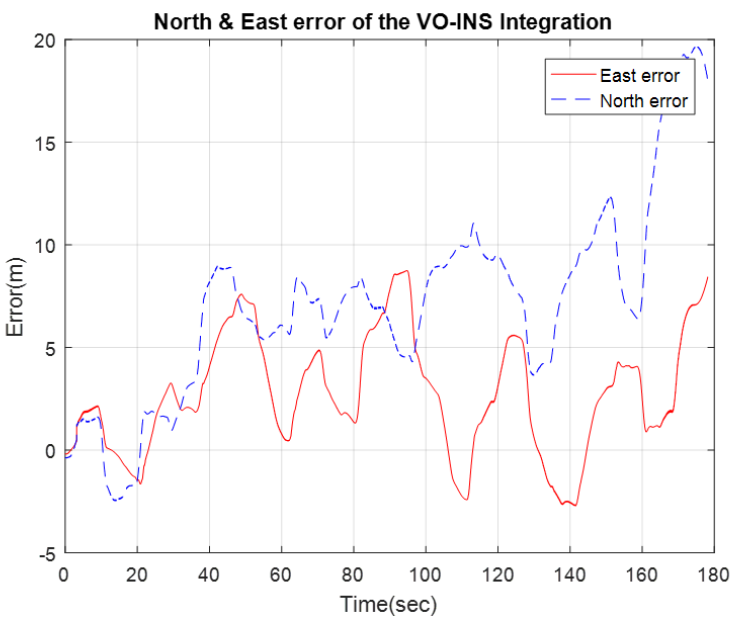

Figure 10. RMSE errors trend in North and East directions for the solution of the camera and INS integration

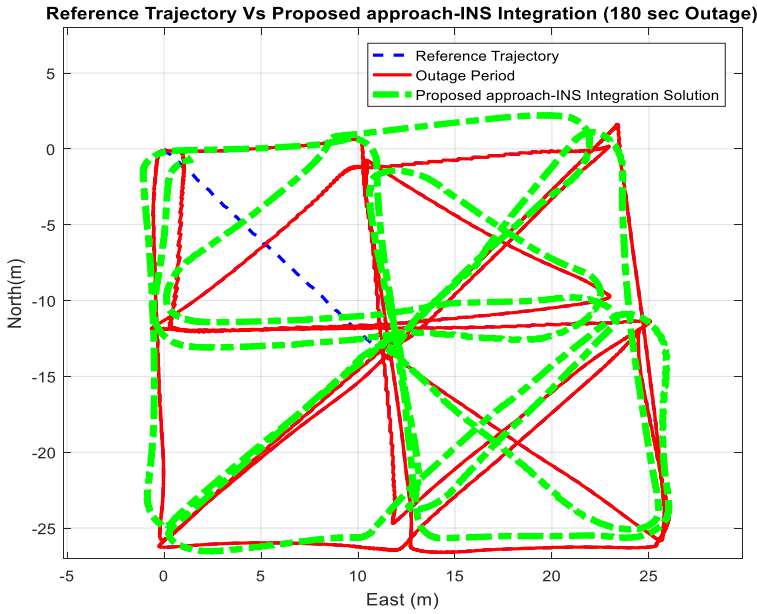

Figure 11. Performance of the integration between the proposed approach (camera with UWB) and INS for 180 seconds of complete GNSS signal outage

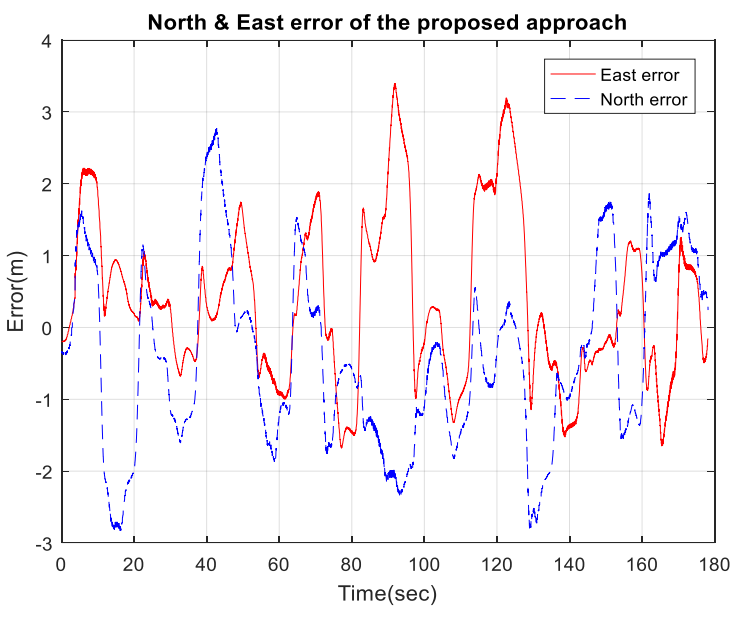

Figure 12. RMSE error trend of the proposed approach (camera and UWB) integrated with INS for a complete 180 seconds of GNSS signal outage 


\begin{tabular}{|l|l|l|l|l|l|}
\hline & \multirow{2}{*}{$\begin{array}{l}\text { UWB } \\
\text {-INS }\end{array}$} & \multirow{2}{*}{$\begin{array}{l}\text { VO } \\
\text {-INS }\end{array}$} & \multirow{2}{*}{$\begin{array}{l}\text { UWB- } \\
\text { VO-INS }\end{array}$} & \multicolumn{2}{|l|}{$\begin{array}{l}\text { Enhancement } \\
\text { from }\end{array}$} \\
\cline { 5 - 6 } & & & & UWB & VO \\
\hline RMSE-N & $1.40 \mathrm{~m}$ & $8.53 \mathrm{~m}$ & $1.34 \mathrm{~m}$ & $4.2 \%$ & $84 \%$ \\
\hline RMSE-E & $1.24 \mathrm{~m}$ & $3.91 \mathrm{~m}$ & $1.20 \mathrm{~m}$ & $3.2 \%$ & $69 \%$ \\
\hline $\begin{array}{l}\text { Max- } \\
\text { Error-N }\end{array}$ & $4.08 \mathrm{~m}$ & $19.6 \mathrm{~m}$ & $2.8 \mathrm{~m}$ & $31 \%$ & $85 \%$ \\
\hline $\begin{array}{l}\text { Max- } \\
\text { Error-E }\end{array}$ & $3.95 \mathrm{~m}$ & $8.75 \mathrm{~m}$ & $3.40 \mathrm{~m}$ & $13 \%$ & $61 \%$ \\
\hline
\end{tabular}

Table 1. The performance of the proposed approach (Camera and UWB integrated with INS) compared against the solution obtained from the UWB system alone and the solution obtained from the camera alone integrated with INS

Table 1 and the previous figures showed that even when using all the beacons of the UWB system alone integrated with INS, or using the camera alone integrated with INS, the integration between the UWB with a camera still enhance the navigation performance when integrated with INS.

\subsection{UWB-Camera-INS Integration (4 Beacons Used the first configuration)}

The following Figures 13 and 14 show the performance of the UWB system alone when fewer anchors are used configuration (f), while the proposed approach performance is shown in Figures 15 and 16 when utilizing less infrastructure by $33 \%$.

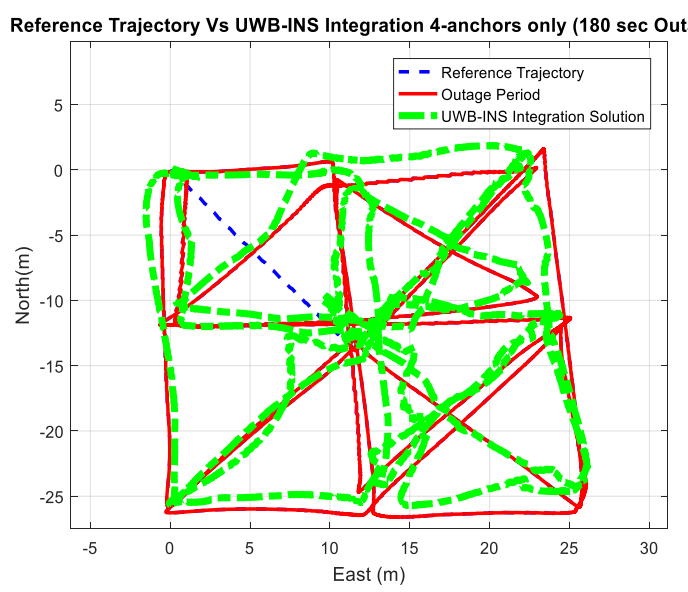

Figure 13. The solution from UWB system alone with lower infrastructure (configuration $\mathrm{f}$ )

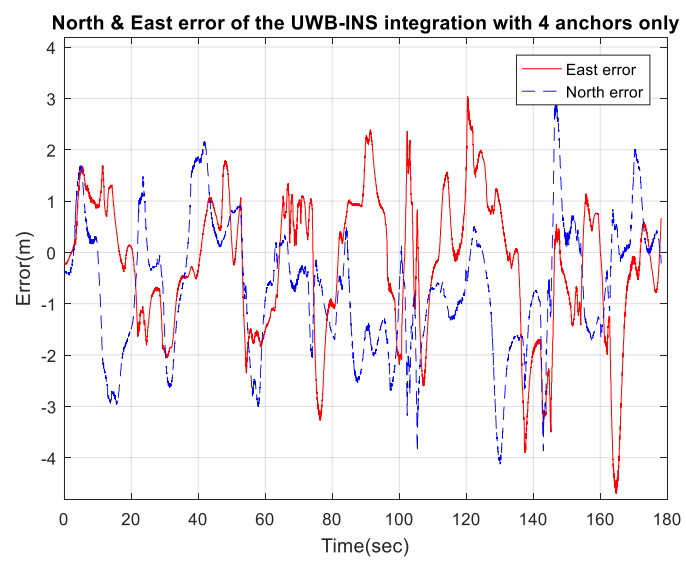

Figure 14. RMSE Errors trend in North and East directions for the UWB system with lower infrastructure utilized (configuration $\mathrm{f}$ )

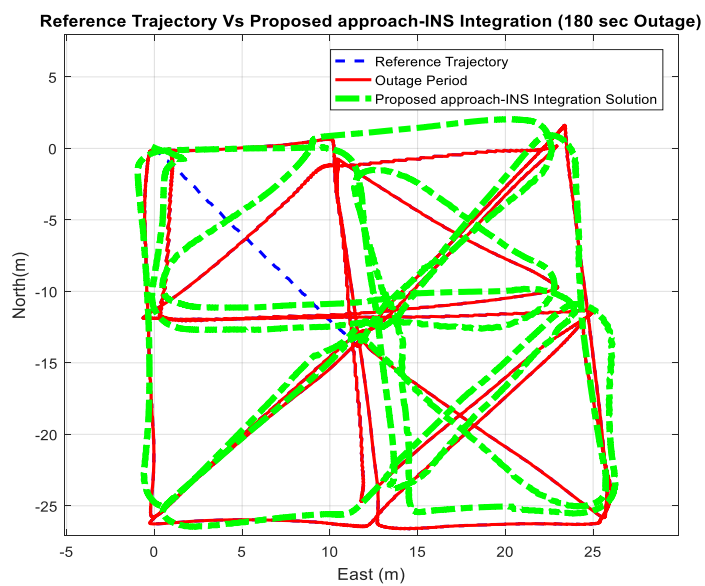

Figure 15. Performance of the proposed approach (camera and

UWB) integrated with INS, with lower UWB system infrastructure for a complete 3-minute GNSS signal outage (configuration $\mathrm{f}$ )

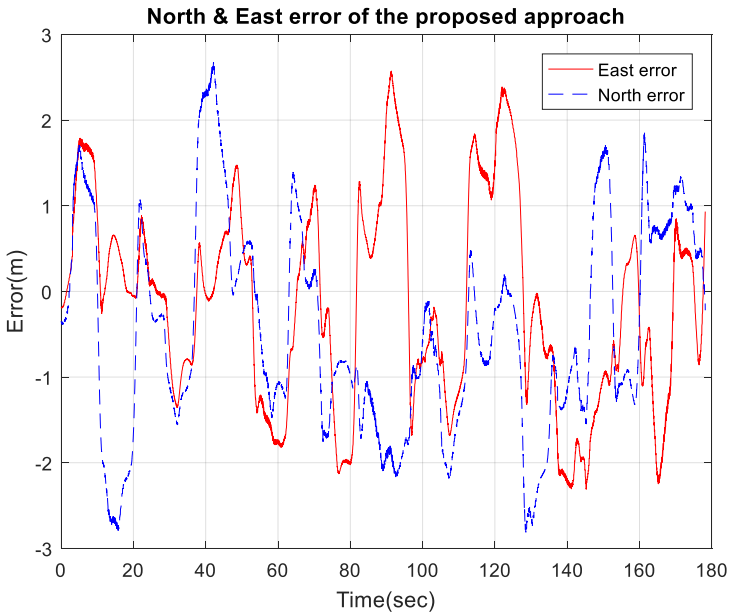

Figure 16. RMSE Errors trend in North and East directions for the proposed approach (camera and UWB) integrated with INS

system with lower infrastructure utilized for a complete 3minute GNSS signal outage (configuration $\mathrm{f}$ ).

\begin{tabular}{|l|c|c|c|}
\hline & $\begin{array}{c}\text { UWB - } \\
\text { INS }\end{array}$ & $\begin{array}{c}\text { UWB- } \\
\text { VO-INS }\end{array}$ & $\begin{array}{c}\text { Enhancement } \\
\text { from UWB }\end{array}$ \\
\hline RMSE-N & $1.44 \mathrm{~m}$ & $1.31 \mathrm{~m}$ & $9 \%$ \\
\hline RMSE-E & $1.36 \mathrm{~m}$ & $1.18 \mathrm{~m}$ & $13 \%$ \\
\hline Max-Error-N & $4.13 \mathrm{~m}$ & $2.82 \mathrm{~m}$ & $31 \%$ \\
\hline Max-Error-E & $4.69 \mathrm{~m}$ & $2.57 \mathrm{~m}$ & $45 \%$ \\
\hline
\end{tabular}

Table 2. The performance of the proposed approach (Camera and UWB integrated with INS) compared against the solution obtained from the UWB system alone (lower infrastructure) and the solution obtained from the camera alone integrated with INS

Shown in the previous Table 2, and figures that the integration between the UWB system with less infrastructure and camera systems leads to improved performance compared to UWB only or camera only integrated with INS.

\subsection{UWB-Camera-INS Integration (4 Beacons Used Second configuration)}

Another experiment with less UWB infrastructure (33\% less) and different configuration (e) to proves the ability of the proposed 
integration in the outdoor environment to enhance the navigation solution during complete GNSS signal outage for 3 minutes.

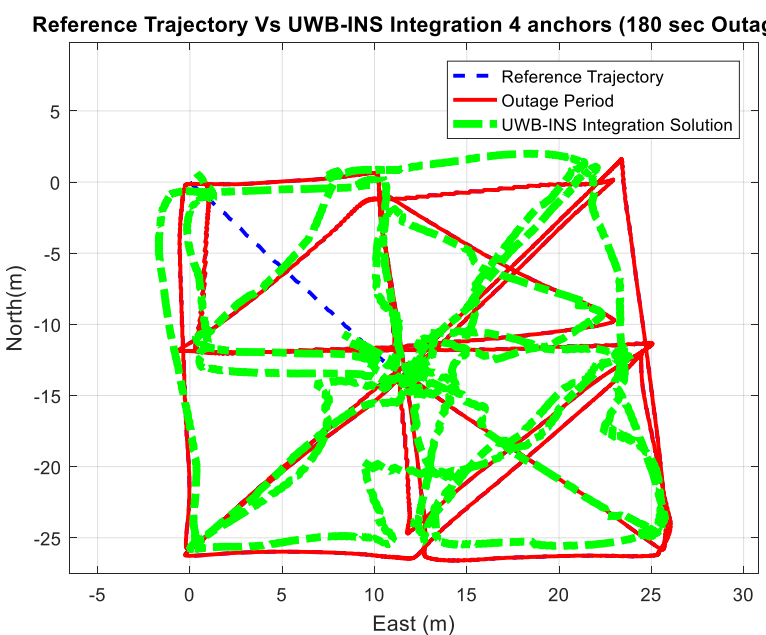

Figure 17. The solution from the UWB system alone with lower infrastructure (configuration e )

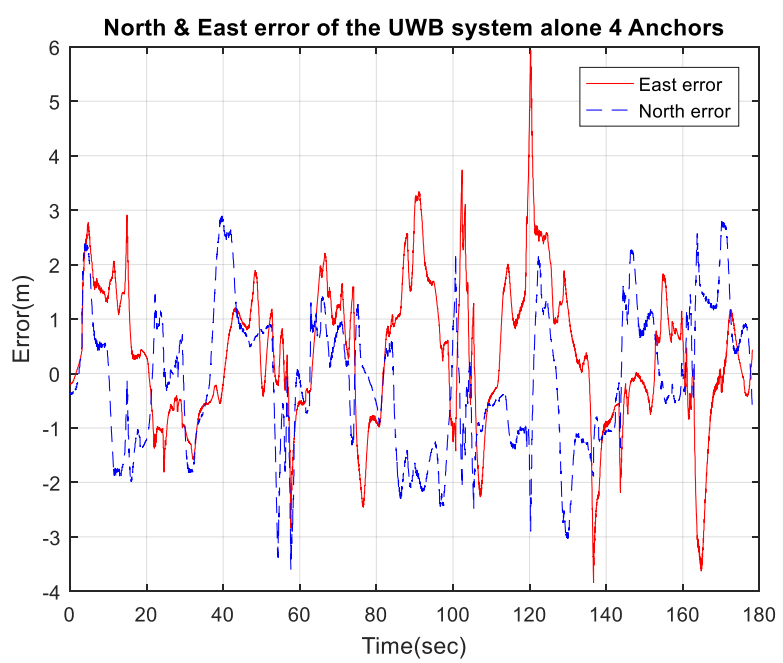

Figure 18. RMSE Errors trend in North and East directions for the UWB system with lower infrastructure utilized (configuration e)

Figures 17 and 18 show the UWB alone performance (second configuration), while Figures 19 and 20 show the performance of the integration between the camera and the UWB system with lower infrastructure by $33 \%$.

\begin{tabular}{|l|c|c|c|}
\hline & $\begin{array}{c}\text { UWB } \\
\text {-INS }\end{array}$ & $\begin{array}{c}\text { UWB- } \\
\text { VO-INS }\end{array}$ & $\begin{array}{c}\text { Enhancement } \\
\text { from UWB }\end{array}$ \\
\hline RMSE-N & $1.27 \mathrm{~m}$ & $1.25 \mathrm{~m}$ & $1.5 \%$ \\
\hline RMSE-E & $1.37 \mathrm{~m}$ & $1.25 \mathrm{~m}$ & $8.7 \%$ \\
\hline Max-Error-N & $3.61 \mathrm{~m}$ & $3.16 \mathrm{~m}$ & $12 \%$ \\
\hline Max-Error-E & $5.93 \mathrm{~m}$ & $3.76 \mathrm{~m}$ & $36 \%$ \\
\hline
\end{tabular}

Table 3. The performance of the proposed approach (Camera and UWB integrated with INS) compared against the solution obtained from the UWB system alone (lower infrastructure) and the solution obtained from the camera alone integrated with INS (Configuration e)

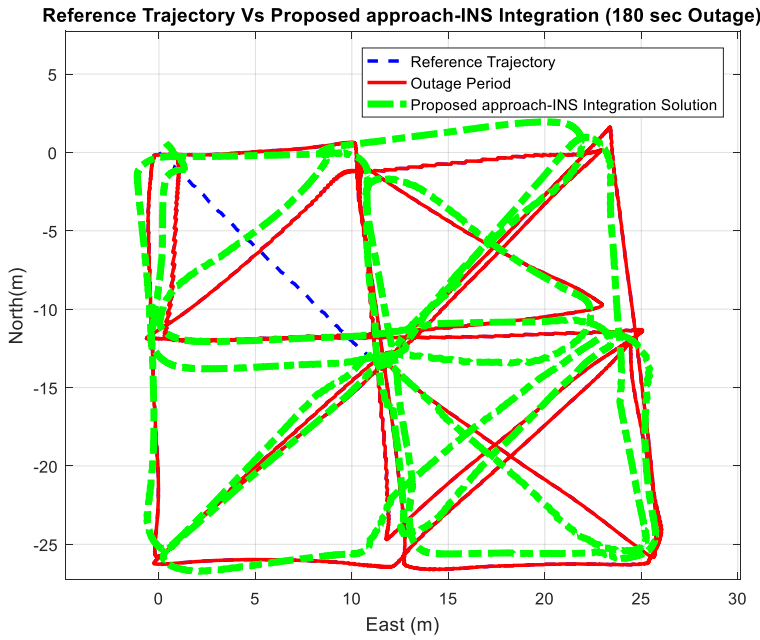

Figure 19. Performance of the proposed approach (camera and

UWB) integrated with INS, with lower UWB system infrastructure for a complete 3-minute GNSS signal outage (configuration e )

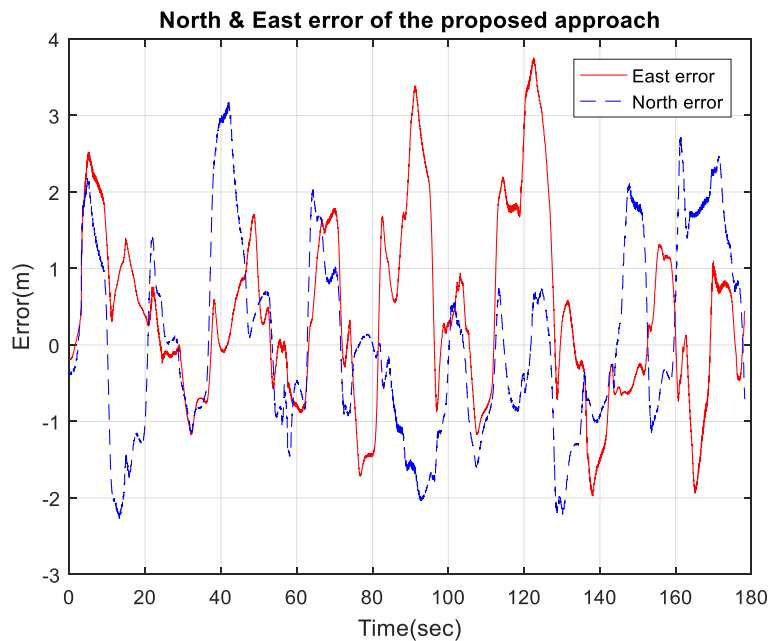

Figure 20. RMSE Errors trend in North and East directions for the proposed approach (camera and UWB) integrated with INS systems with lower infrastructure utilized for a complete 3minute GNSS signal outage (configuration e)

This experiment also proved through the results shown in Table 3, and previous figures, the ability of the integration between camera and UWB systems to enhance the navigation performance of UAVs during GNSS signal outage compared to UWB system only with fewer numbers of anchors utilized to estimate the drone positions.

Table 4 shows the results of the proposed approach for all the configuration shown in Figure (5), which represents the UWB with less infrastructure. The results shown in this table proves that adopting such configuration enhance the navigation system with less UWB infrastructure to reduce the system overall cost. 


\begin{tabular}{|l|c|c|c|c|c|c|c|c|c|c|c|c|}
\hline & $\begin{array}{c}\text { UW } \\
\text { B (a) }\end{array}$ & $\begin{array}{c}\text { UWB } \\
\text { \& VO } \\
(\mathrm{a})\end{array}$ & $\begin{array}{c}\text { UWB } \\
(\mathrm{b})\end{array}$ & $\begin{array}{c}\text { UWB } \\
\text { \& VO } \\
\text { (b) }\end{array}$ & $\begin{array}{c}\text { UWB } \\
(\mathrm{c})\end{array}$ & $\begin{array}{c}\text { UWB } \\
\text { \& VO } \\
(\mathrm{c})\end{array}$ & $\begin{array}{c}\text { UWB } \\
(\mathrm{d})\end{array}$ & $\begin{array}{c}\text { UWB } \\
\text { \& VO } \\
(\mathrm{d})\end{array}$ & $\begin{array}{c}\text { UWB } \\
(\mathrm{e})\end{array}$ & $\begin{array}{c}\text { UWB } \\
\& \text { VO } \\
(\mathrm{e})\end{array}$ & $\begin{array}{c}\text { UWB } \\
(\mathrm{f})\end{array}$ & $\begin{array}{c}\text { UWB } \\
\& \text { VO } \\
(\mathrm{f})\end{array}$ \\
\hline RMSE-N & 1.48 & 1.35 & 1.86 & 1.64 & 1.45 & 1.37 & 1.49 & 1.37 & 1.27 & 1.25 & 1.44 & 1.31 \\
\hline Max-E-N & 4.48 & 3.01 & 5.66 & 3.78 & 4.23 & 3.56 & 7.31 & 3.21 & 3.61 & 3.16 & 4.13 & 2.82 \\
\hline RMSE-E & 1.68 & 1.63 & 1.47 & 1.24 & 1.25 & 1.22 & 1.23 & 1.12 & 1.37 & 1.25 & 1.36 & 1.18 \\
\hline Max-E-E & 5.03 & 4.36 & 6.72 & 3.40 & 5.59 & 3.38 & 4.02 & 3.03 & 5.93 & 3.76 & 4.69 & 2.57 \\
\hline
\end{tabular}

Table 4. The performance of the proposed approach in meters for all the sample configuration shown in Figure (5)

\section{CONCLUSION}

Small/commercial drones mainly depends on the integration between GNSS system and INS system to navigate for longer periods of time, while due to the challenges that may face the drone during its missions, that may lead to GNSS signals blockage/outage, the navigation performance will be massively deteriorated specially when the used IMU is a MEMS-based lowcost, which exhibits massive accumulation of errors. Other aiding system/sensors must be utilized for that purpose. Ultra-wideband system is considered a good candidate during GNSS outage period, due to its low weight, small size, and low power consumption, but UWB is mainly used for indoor terrestrial applications, as it requires a large infrastructure of UWB anchors to cover a large area in the outdoor environment. The monocular camera also is one of the mainly used GNSS replacement systems during GNSS outage period, due to its adequate size, weight, and power consumption. But cameras are sensitive to environmental changes, and lack of detected features may affect its performance. Also integrating camera measurements as velocity with INS through EKF suffers of heading drift. This paper showed that the integration between UWB and camera systems with variable EKF uncertainty scheme. In our experiments, even after decreasing the UWB infrastructure by 33\%, the performance of the proposed approach is still better than both that provided by the integration of the full UWB network with INS, and that of camera - INS integration. The proposed approach was evaluated with a real flight, using 3DR solo quadcopter equipped with low-cost IMU (MPU-9250), camera (GoPro hero4) and UWB system (Pozyx). The results of the proposed approach during a complete 180 secs of GNSS signal outage lead to a RMSE error of $1.2 \mathrm{~m}$ in both North and East directions, even when considering a reduced size of the UWB network.

\section{REFERENCES}

Bay, H., Tuytelaars, T., Van Gool, L., 2006. SURF: Speeded Up Robust Features, in: Leonardis, A., Bischof, H., Pinz, A. (Eds.), Computer Vision - ECCV 2006, Lecture Notes in Computer Science. Springer Berlin Heidelberg, pp. 404-417.

Chuanqi, C., Xiangyang, H., Zhenjie, Z., Mandan, Z., 2017. Monocular visual odometry based on optical flow and feature matching, in: 2017 29th Chinese Control And Decision Conference (CCDC). Presented at the 2017 29th Chinese Control And Decision Conference (CCDC), pp. 4554-4558. https://doi.org/10.1109/CCDC.2017.7979301

Eynard, D., Vasseur, P., Demonceaux, C., Frémont, V., 2010. UAV altitude estimation by mixed stereoscopic vision, in: 2010 IEEE/RSJ International Conference on Intelligent Robots and Systems. Presented at the 2010 IEEE/RSJ International Conference on Intelligent Robots and Systems, pp. 646-651. https://doi.org/10.1109/IROS.2010.5652254

Heinrich, A., 2017. An Optical Flow Odometry Sensor Based on the Raspberry Pi Computer [WWW Document]. URL/paper/AnOptical-Flow-Odometry-Sensor-Based-on-the-Pi-
Heinrich/310fa5278f9e05383ed46ffd74ddc36bbacc47a9 Johnson, A.E., Goldberg, S.B., Cheng, Y., Matthies, L.H., 2008. Robust and Efficient Stereo Feature Tracking for Visual Odometry. 2008 IEEE Int. Conf. Robot. Autom. 39-46. https://doi.org/10.1109/ROBOT.2008.4543184

Kong, W., Zhang, D., Zhang, J., 2015. A ground-based multisensor system for autonomous landing of a fixed wing UAV, in: 2015 IEEE International Conference on Robotics and Biomimetics (ROBIO). Presented at the 2015 IEEE International Conference on Robotics and Biomimetics (ROBIO), pp. 13031310. https://doi.org/10.1109/ROBIO.2015.7418951

Mansur, S., Habib, M., Pratama, G.N.P., Cahyadi, A.I., Ardiyanto, I., 2017. Real time monocular visual odometry using Optical Flow: Study on navigation of quadrotors UAV, in: 2017 3rd International Conference on Science and Technology Computer (ICST). Presented at the 2017 3rd International Conference on Science and Technology - Computer (ICST), pp. 122-126. https://doi.org/10.1109/ICSTC.2017.8011864

Mostafa, M.M., Moussa, A.M., El-Sheimy, Naser, Sesay, Abu B., "Optical Flow Based Approach for Vision Aided Inertial Navigation Using Regression Trees," Proceedings of the 2017 International Technical Meeting of The Institute of Navigation, Monterey, California, January 2017, pp. 856-865. doi:10.33012/2017.14898

Nistér, D., Naroditsky, O., Bergen, J., 2004. Visual odometry, in: Computer Vision and Pattern Recognition, 2004. CVPR 2004. Proceedings of the 2004 IEEE Computer Society Conference On. Ieee, pp. I-I.

Noureldin, A., Karamat, T.B., Georgy, J., 2013. Fundamentals of Inertial Navigation, Satellite-based Positioning and their Integration. Springer Berlin Heidelberg, Berlin, Heidelberg. https://doi.org/10.1007/978-3-642-30466-8

Tiemann, J., Schweikowski, F., Wietfeld, C., 2015. Design of an UWB indoor-positioning system for UAV navigation in GNSSdenied environments, in: 2015 International Conference on Indoor Positioning and Indoor Navigation (IPIN). Presented at the 2015 International Conference on Indoor Positioning and Indoor Navigation (IPIN). doi:10.1109/IPIN.2015.7346960

Tiemann, J., Wietfeld, C., 2017. Scalable and precise multi-UAV indoor navigation using TDOA-based UWB localization, in: 2017 International Conference on Indoor Positioning and Indoor Navigation (IPIN). Presented at the 2017 International Conference on Indoor Positioning and Indoor Navigation (IPIN), pp. 1-7. https://doi.org/10.1109/IPIN.2017.8115937

Yousif, K., Bab-Hadiashar, A., Hoseinnezhad, R., 2015. An Overview to Visual Odometry and Visual SLAM: Applications to Mobile Robotics. Intell. Ind. Syst. 1, 289-311. https://doi.org/10.1007/s40903-015-0032-7 